\title{
EDITORIAL
}

\section{The dark side of antituberculosis therapy: adverse events involving liver function}

\author{
T. Schaberg
}

The World Health Organization estimated that 1.7 billion people worldwide were infected with Mycobacterium tuberculosis in 1990. The number of new cases in the same year was estimated to be as high as 8 million, with a death toll of approximately 3 million [1]. During the nineties, these figures are expected to increase to more than 10 million new cases and 3.6 million deaths each year. Thus, tuberculosis will still remain the leading cause of death in the world from a single infectious disease at the beginning of the new century. Although there has been a remarkable decrease in morbidity and mortality in the developed countries since 1900, tuberculosis rapidly spread to the developing countries during the last 95 yrs despite all efforts to control the disease. Today, we are far away from any hope of eradication of "the captain of all these men of death" [2]. Due to two factors, the decrease of tuberculosis in Europe and the USA during the last 100 yrs and the remarkable achievements of modern antituberculosis chemotherapy [3], a "wealth of ignorance" regarding our understanding of the pathogenesis of tuberculosis emerged during the seventies and research activities on new substances for treating tuberculosis stopped [2]. However, the problem of tuberculosis has attracted new attention from the medical community, since there has been an increase in the incidence of tuberculosis in developed countries the late eighties, which was first observed in large urban areas, such as New York City [4], but, thereafter, also in nearly all European countries and other parts of the USA [5].

Based on the two principles of "case-finding" and chemotherapy, one should assume that the control of tuberculosis would be easy once the problem of tuberculosis is recognized. However, although modern chemotherapy is extremely efficient in controlled studies, success in the field is considerably lower. In recent years, in particular, two major treatment problems have gained attention: firstly, the development of drug resistance; and, secondly, the tolerance of the substances used.

Since the rate of natural resistance of $M$. tuberculosis against major drugs, such as isoniazid, rifampin and pyrazinamide, is very low (approximately one resistant bacterium out of a population of $10^{8}$ bacteria), most of the observed resistance of $M$. tuberculosis is "man-made". Poor compliance and nonadherance to the prescribed therapy, as well as failures of drug prescription and therapy-monitoring,

Section of Infectious Diseases and Immunology, Dept of Pulmonary Medicine, Chest Hospital Heckeshorn-Zehlendorf, Zum Heckeshorn 33, D-14109 Berlin-Wannsee, Germany. are responsible for most cases of acquired resistance. This can easily be demonstrated by the fact that the most important risk factor for the occurrence of drug-resistance in $M$. tuberculosis is prior treatment $[6,7]$. Only in areas where a high rate of acquired resistance is already endemic will new infections with resistant strains contribute substantially to the total resistance problem.

Tolerance of antituberculosis therapy is the other major problem of tuberculosis treatment today. This edition of the Journal contains a review article dealing with the common problem of hepatotoxicity as well as a sideeffect of antituberculosis therapy [8]. Based on their own experience with three cases of fatal hepatic complications during antituberculosis treatment, the authors reviewed the literature and developed a flow-sheet for the management of hepatotoxicity during antituberculosis therapy. For two reasons, the article by THOMPSON et al. [8] is a significant contribution. Firstly, the authors give concrete recommendations for the management both of patients with tuberculosis who are at risk for hepatotoxicity and those who have already developed hepatotoxicity during antituberculosis therapy. Secondly, the paper brings to our attention a well-known problem that has not yet been sufficiently solved.

The three most important drugs used for treating tuberculosis are hepatotoxic [9, 10]. For single substances, it has been shown that toxicity is primarily dose-dependant [11]. The incidence of hepatic toxicity during the intake of isoniazid alone is well-known from experience with this drug in preventive chemotherapy [12]. At a dose of $5 \mathrm{mg} \cdot \mathrm{kg}^{-1}$, the frequency of clinical hepatitis is $0.5-3 \%$, with a frequency of raised transaminases of $10-25 \%$ [13]. For rifampin $\left(10 \mathrm{mg} \cdot \mathrm{kg}^{-1}\right)$, clinically apparent hepatotoxicity has been reported to occur in $2-5 \%$ of cases and altered liver function tests in 10-15\% [13]. Elevated transaminases following therapy with pyrazinamide $\left(25-30 \mathrm{mg} \cdot \mathrm{kg}^{-1}\right)$ have been observed in $10 \%$ of patients, whereas the frequency of hepatitis has been found to be lower than 5\% [8]. However, following the principles of modern combined chemotherapy, the combination of all three drugs mentioned above are considered as the gold standard therapy [14]. Surprisingly, although there is a large body of evidence for additive hepatotoxicity both in animals and in humans, the incidence of severe adverse effects related to the three major drugs was shown to be quite low in meta-analysis [13], and in most recently published controlled studies (table 1). Regarding hepatotoxicity, most studies report only 
the frequency of hepatitis but not that of elevated transaminases or disturbed liver function tests. Therefore, analysis of total hepatotoxicity is difficult.

A second problem arising in the analysis of hepatotoxicity is the population in which the different studies were performed. One can assume that the frequency of some risk factors for hepatotoxicity (e.g. alcohol abuse, i.v. drug abuse, diabetes mellitus, or concomitant intake of other hepatotoxic drugs) is lower in developing countries than in the urban population at risk for tuberculosis in developed countries, where, on the other hand, the rate of viral hepatitis might be lower than in Africa or Asia. Moreover, it is well-known that chemotherapy in patients with human immunodeficiency virus (HIV) infection is associated with more adverse effects than therapy in HIV-negative patients. For example, in a recent study by Small et al. [21] 7 of 132 (5.3\%) HIV-positive patients with tuberculosis developed severe hepatotoxicity during chemotherapy but none of the HIV-negative control patients with tuberculosis.

In my opinion, there are two aspects to the problem of hepatotoxicity from antituberculosis drugs. One is the direct risk of a patient to develop severe hepatitis. This risk can be easily diminished if the patient is monitored in an appropriate manner, and any rise in transaminases or changes in liver function tests induce frequent laboratory monitoring. The other side of the problem, which has not been addressed in studies so far, is that hepatotoxicity may lead to a withdrawal of one of the basic substances. A patient who does not receive a combination of isoniazid and rifampin over 6-9 months, supplemented with pyrazinamide during the first 8 weeks of treatment, does not receive the best treatment available and is at risk of a relevant relapse rate. Moreover, the total duration of treatment is prolonged in such cases, because treatment without isoniazid or pyrazinamide must be continued for 9-12 months and treatment without rifampin for 12 months [14]. Even more complicated is the situation when hepatotoxicity contributes towards a further limitation of treatment possibilities in patients with single or multidrug-resistant tuberculosis. Although we do not have much data on this problem, it seems justified to give it some consideration. Our preliminary observation of 200 consecutive tuberculosis therapies in patients from an inner city hospital in 1993/1994 identified 33

Table 1. - Hepatotoxicity in recent controlled studies of antituberculosis chemotherapy including isoniazid, rifampin and pyraziamide ( $n=3,506$ parients)

\begin{tabular}{rrrccc}
\hline [Ref.] & Year & $\begin{array}{c}\text { Pts } \\
\mathrm{n}\end{array}$ & $\begin{array}{c}\text { Hepatitis } \\
\%\end{array}$ & $\begin{array}{c}\text { Raised } \\
\text { transaminases } \\
\%\end{array}$ & $\begin{array}{c}\% \text { drug(s) } \\
\text { terminated* } \\
\%\end{array}$ \\
\hline$[15]$ & 1981 & 330 & 3.0 & - & 3.0 \\
{$[16]$} & 1982 & 1027 & 2.0 & 30.5 & 2.0 \\
{$[17]$} & 1984 & 213 & 2.8 & 13.6 & 2.8 \\
{$[18]$} & 1991 & 240 & 2.5 & 2.6 & 3.3 \\
{$[19]$} & 1991 & 1386 & 2.3 & - & 1.6 \\
{$[20]$} & 1991 & 310 & 0.6 & - & 0.6 \\
\hline
\end{tabular}

*: terminated because of hepatotoxicity. Pts: patients; [Ref.]: reference number.
(16.5\%) cases of hepatotoxicity which necessitated interruption of therapy, and $22(11 \%)$ cases in which one or more of the three standard drugs had to be terminated permanently because of hepatotoxicity (T. Schaberg, unpublished data). If we consider together the patients with single drug resistance $(5.8 \%)$ and those with multidrug resistance $(5.8 \%)$ [7], it emerges, in fact, that nearly $22 \%$ of the tuberculosis patients could not receive the best possible treatment. One conclusion to be drawn from this scenario, besides the need for intense monitoring of patients on antituberculosis therapy to avoid severe hepatotoxicity, is that new substances with lower hepatotoxicity are urgently needed in order to control tuberculosis in our communities appropriately.

Some suggestions can be made for future research activities and clinical practice. Regarding research in this area, new substances which are effective against $M$. tuberculosis without serious adverse effects are definitely needed. Therefore, pharmaceutical companies are urgently asked to develop new compounds, which can then be tested in clinical trials. Since clinical trials investigating new substances require a follow-up of at least 2 yrs, the total time necessary to complete such a study would be $5 \mathrm{yrs}$ or more. It is, therefore, necessary to take into account other approaches for assessing the efficacy of a new substance. Two recently developed methods seem to be suitable for this purpose. One is the assessment of the bactericidal activity of a substance by quantitative culturing of specimens from highly smear-positive cases during the first week of treatment with monotherapy [22]. The other is to determine the sterilizing properties of a new substance by examining culture conversion after 2 months of therapy, since it has been shown that there is a close correlation between the rate of culture conversion and relapse 2-5 yrs after chemotherapy [23].

Although the principles of antituberculosis therapy have been determined and published in the last $40 \mathrm{yrs}$, the practice of this therapy is still based on vast experience and profound knowledge of the wide range of possible side-effects of the substances used. Therefore, it seems appropriate to demand that antituberculosis therapy should be performed only by thoroughly trained and experienced doctors. Monitoring for possible adverse effects, such as hepatotoxicity, especially in patients at risk, is part of good clinical practice and should be carried out routinely. In any case, death from antituberculosis therapy should be a extremely rare complication today, because the art of tuberculosis therapy is to cure the patients and not to merely prescribe drugs.

\section{References}

1. Kochi A. The global tuberculosis situation and the new control strategy of the World Health Organization. Tubercle 1991; 72: 1-6.

2. Bloom BR, Murray CJL. Tuberculosis: commentary on a re-emergent killer. Science 1992; 257: 1055-1064.

3. Iseman MD, Sbarbaro JA. Short-course chemotherapy of tuberculosis. Hail Britannia (and friends)! Am Rev Respir Dis 1991; 143: 697-698. 
4. Reichman LB. The U-shaped curve of concern. Am Rev Respir Dis 1991; 144: 741-742.

5. Styblo K. The impact of HIV infection on the global epidemiology of tuberculosis. Bull Int Union Tuberc Lung Dis 1991; 66: 27-32.

6. Frieden TR, Sterling T, Pablos-Mendez A, et al. The emergence of drug-resistant tuberculosis in New York City. N Engl J Med 1993; 328: 521-526.

7. Schaberg T, Gloger G, Reichert B, et al. Drug-resistant pulmonary tuberculosis in Berlin, Germany 1987-1993. Eur Respir J 1995; 8: 278-284.

8. Thompson NP, Caplin ME, Hamilton MI, et al. Antituberculosis medication and the liver: dangers and recommendations in management. Eur Respir $J$ 1995; 8: 1384-1388.

9. Girling DJ. Adverse effects of antituberculosis drugs. Drugs 1992; 23: 56-74.

10. Westphal JF, Vetter D, Brogard JM. Hepatic side effects of antibiotics. J Antimicrob Chemother 1994; 33: $387-$ 401.

11. Bartmann K. Antituberculosis drugs. Berlin, Heidelberg, New York, Springer Verlag, 1988.

12. Jordan TJ, Lewitt EM, Reichman LB. Isoniazid preventive therapy for tuberculosis. Am Rev Respir Dis 1991; 144: 1357-1360.

13. Steele MA, Burk RF, DesPrez RM. Toxic hepatitis with isoniazid and rifampin. Chest 1991; 99: 465-471.

14. American Thoracic Society. Treatment of tuberculosis and tuberculosis infection in adults and children. Am J Respir Crit Care Med 1994; 149: 1359-1374.

15. Singapore Tuberculosis Service - British Medical Resarch Council. Clinical trial of six month and four month regimens of chemotherapt in the treatment of pulmonary tuberculosis: the results up to 30 months. Tubercule 1981; 62; 95-102.

16. Hong Kong Chest Service - British Medical Research Council. Controlled trial of 4 three-times-weekly regimens and a daily regimen all given for 6 months for pulmonary tuberculosis. Second report: the results up to 24 months. Tubercle 1982; 63: 89-98.

17. Snider DE, Graczyk J, Bek E, et al. Supervised six month treatment of newly diagnosed pulmonary tuberculosis using isoniazid, rifampin, and pyrazinamide with and without streptomycin. Am Rev Respir Dis 1984; 130: 1091-1094.

18. Hong Kong Chest Service - Tuberculosis Research Centre Madras - British Medical Research Council. A controlled clinical comparison of 6 and 8 months of antituberculosis chemotherapy in the treatment of patients with silicotuberculosis in Hong Kong. Am Rev Respir Dis 1991; 143: 262-267.

19. Hong Kong Chest Service - British Medical Research Council. Controlled trial of 2, 4 and 6 months of pyrazinamide in 6 month, three times weekly regimens for smear-positive pulmonary tuberculosis, including an assessment of a combined preparation of isoniazid, rifampin and pyrazinamide. Am Rev Respir Dis 1991; 143: 700-706.

20. Singapore Tuberculosis Service - British Medical Research Council. Assessment of a daily combined preparation of isoniazid, rifampin and pyrazinamide in a controlled trial of three 6 month regimens for smear-positive pulmonary tuberculosis. Am Rev Respir Dis 1991; 143: 707-712.

21. Small PM, Schecter GF, Goodman PC, et al. Treatment of tuberculosis in patients with advanced human immunodeficiency virus infection. $N$ Engl J Med 1991; 324: 289-294.

22. Sirgel FA, Botha FJJ, Parkin DP, et al. The early bactericidal activity of rifabutin in patients with pulmonary tuberculoisis measured by sputum viable counts: a new method of drug assessment. $J$ Antimicrob Chemother 1993; 32: 867-875.

23. Mitchison DA. Assessment of new sterilizing drugs for treating pulmonary tuberculosis by culture at 2 months. Am Rev Respir Dis 1993; 147: 1062-1063. 